\title{
Perfil dos estudantes que ingressam no curso de Odontologia: motivos da escolha
}

\author{
Maria do Socorro Silva Ferreira Mendes*; Mônica Peluzio Benevides Valente*; Ellen Camargo \\ Rodrigues*; Josiane Aleixo da Silva Siqueira*; Elvis Bruno Almeida da Silva*; Neide Cristina \\ Nascimento Santos*; Flávia Martão Flório**; Luciane Zanin Souza**; Arlete Maria Gomes Oliveira** \\ * Estudante, Mestrado em Saúde Coletiva, Faculdade São \\ Leopoldo Mandic \\ ** Docente, Departamento de Saúde Coletiva, Faculdade São \\ Leopoldo Mandic
}

Recebido em 16/02/2018. Aprovado em 19/12/2018.

\begin{abstract}
RESUMO
O objetivo deste estudo, com recorte transversal de natureza quantitativa, foi avaliar o perfil, a percepção e o motivo da escolha pelo curso de Odontologia em uma faculdade particular de Campinas (SP). Do total de 153 alunos matriculados em 2016, 102 (66,7\%) aceitaram participar da pesquisa. O instrumento de pesquisa foi um questionário estruturado, autoaplicável, com 22 questões que versavam sobre perfil socioeconômico, demográfico, percepção sobre o curso e os motivos de sua escolha. Entre os respondentes, 81,4\% eram do sexo feminino. A faixa etária de 17 a 20 anos predominou $(67,3 \%)$, sendo a maioria solteiros $(89,3 \%)$, tendo cursado ensino médio em escola particular $(80,4 \%)$, com renda familiar acima de $\mathrm{R} \$ 8.800,00(54,0 \%)$. Quando abordados sobre o motivo da escolha pelo curso, $61,7 \%$ optaram por ser da área da saúde; e 29,4\% sofreram influência de um dentista. Quanto à pretensão profissional, 6,8\% querem trabalhar somente no setor público, $47,0 \%$ querem ser assalariados e montar seu próprio negócio e 38\% querem trabalhar de forma autônoma em consultório. Sobre o curso, $62,8 \%$ se declararam satisfeitos com o corpo docente; $72,5 \%$, com a matriz curricular, e a maioria $(70,5 \%)$ deseja cursar especialização após a graduação. Sobre a escolha, 97,0\% estão satisfeitos; e 96,0\% se sentem preparados para enfrentar o mercado de trabalho. A percepção dos respondentes é positiva quanto à formação. Há o reconhecimento da tendência de assalariamento na profissão, embora demonstrem o desejo de atuação no setor privado. Descritores: Odontologia. Escolha da profissão. Estudantes.
\end{abstract}

\section{INTRODUÇÃO}

$\mathrm{O}$ processo de escolha profissional costuma ser difícil e cercado de angústias, dentre as quais destacam-se as inseguranças com a escolha, as diversificadas opções de cursos, aspectos socioeconômicos e culturais relacionados com a profissão, mercado de trabalho e concorrido processo seletivo instituído pelas universidades ${ }^{1}$.

Assim como toda profissão, a Odontologia também possui particularidades que necessitam de explicação, visando permitir o entendimento do seu atual posicionamento, bem como a compreensão e 
elucidação de suas dificuldades ${ }^{2}$. Nos últimos anos, observa-se que houve um interesse maior pelo curso devido à incorporação da equipe de saúde bucal na Estratégia Saúde da Família (ESF), pois esse quadro vem seguido de desafios para a adequação da formação para o trabalho, em conformidade com os princípios do Sistema Único de Saúde (SUS) e da necessidade de gerar uma melhor disposição de profissionais entre as regiões brasileiras ${ }^{3}$.

Considerando a trajetória histórica da Odontologia no Brasil, podem-se constatar transformações que vão desde a concepção do seu exercício como arte de cuidar dos dentes até a aquisição da autonomia enquanto ciência ministrada por instituições escolares ${ }^{4}$.

A motivação e a identificação profissional podem advir da história pessoal e familiar de cada estudante do curso, podendo estas ser explicadas pelo contato frequente com a profissão desde a infância. De forma semelhante, quando o aluno adentra no curso, a motivação torna-se evidente devido à aprovação no vestibular e ao ingresso em uma faculdade, contudo, ao longo dos semestres, o acadêmico pode se sentir desmotivado e pensar em outra escolha profissional ${ }^{5}$.

A percepção sobre o curso de Odontologia no que se refere ao sucesso e status elitista de profissionais da área, bem como as possibilidades de não comprometimento social enquanto profissionais de saúde, tem sido uma das vantagens que vêm motivando a escolha pelo curso há anos ${ }^{6}$.

A instituição de ensino tem forte influência na adesão e permanência do aluno, pois, durante a graduação, o acadêmico passa por etapas que podem consolidar ou não sua escolha profissional ${ }^{7}$.

Há uma nova adequação quanto à realidade do mercado e da população, a partir da atual e nova formação profissional do cirurgião-dentista. As Diretrizes Curriculares Nacionais para o ensino de graduação em Odontologia ditam que o profissional da área deve zelar pela atenção integral a seus pacientes, enfocando o atendimento ético, justo e humano, independentemente do mercado no qual está inserido, público ou privado ${ }^{8}$.

A faculdade tem como missão preparar um profissional crítico e reflexivo, cultivando técnicas pedagógicas, que posteriormente o auxiliará em sua inserção no mercado de trabalho com um perfil ético e responsável ${ }^{9}$. Em contrapartida, os anseios pela carreira profissional, o modo como os discentes conduzem seus estudos no período acadêmico, como direcionam seus relacionamentos interpessoais e como exercem seus papéis na sociedade atrapalham o acadêmico ter uma visão determinante ${ }^{6}$.

De acordo com dados do Ministério da Educação e Cultura (MEC) ${ }^{10}$ existem, atualmente, 451 cursos de Odontologia no Brasil. A maior concentração está na região Sudeste (totalizando 98), distribuídos nos estados de Minas Gerais (30), do Espírito Santo (11), do Rio de Janeiro (27) e de São Paulo (30).

O acadêmico, ao encerrar o curso de Odontologia, leva consigo expectativas da instituição que o formou, a qual deseja que este seja um profissional capacitado a exercer atividades referentes tanto ao escopo técnico/científico quanto à saúde bucal da população, pautado em princípios éticos e legais ${ }^{11}$.

Nesse contexto, o objetivo desta pesquisa foi avaliar o perfil, a percepção e o motivo da escolha pelo curso de Odontologia de uma instituição privada, bem como a escolha profissional, evidenciando as influências sociais e pessoais que originam essa opção.

\section{METODOLOGIA}

Trata-se de estudo observacional transversal. A pesquisa se desenvolveu em uma instituição privada, sediada em Campinas, estado de São Paulo. A população do estudo foi composta por todos os alunos ( $n=153$ ) do Curso de Odontologia, de ambos os sexos, regularmente matriculados no ano de 2016. 
Foram excluídos da pesquisa os acadêmicos que não estavam presentes no dia da aplicação do questionário, visitantes, de outras universidades em situação de intercâmbio e em período de transferência, finalizando uma amostra de 102 estudantes.

Para a coleta dos dados, utilizou-se um questionário semiestruturado, validado no estudo de Junqueira et al. ${ }^{12}$, contendo 22 questões de múltipla escolha e 2 questões subjetivas pertinentes ao comportamento do aluno, perfil e motivos da escolha pelo curso de Odontologia.

Previamente à coleta de dados, realizou-se um treinamento com os pesquisadores para melhor conhecimento do instrumento da pesquisa, com o intuito de obter resultados confiáveis, bem como de verificar possíveis falhas metodológicas. A pesquisa ocorreu em cinco dias consecutivos do mês de agosto de 2016, durante os dois períodos do dia (manhã e tarde). Os alunos foram abordados em sala de aula, sendo fornecidas explicações sobre a pesquisa e, em seguida, entregue o Termo de Consentimento Livre e Esclarecido - TCLE. Os questionários foram entregues aos alunos que aceitaram participar da pesquisa, resguardado o tempo de 10 a 15 minutos para as respostas. Os instrumentos foram aplicados sem a intervenção do pesquisador, porém, estando o mesmo à disposição para qualquer esclarecimento. Os dados obtidos foram inseridos e organizados no programa Microsoft Excel ${ }^{\circledR}$ (MS-Excel v. 16.0), a fim de obter as frequências relativas e absolutas das respostas de cada questão. Para análise estatística, foram utilizados os testes Qui-Quadrado, exato de Fischer e Odss ratio. O nível de significância adotado na decisão dos testes estatísticos foi de $5 \%$ e Intervalo de Confiança (IC) de $95 \%$.

\section{RESULTADOS}

A primeira parte do questionário envolveu os dados referentes às variáveis socioeconômicas dos acadêmicos entrevistados. Houve um número bem expressivo do sexo feminino, totalizando $81,4 \%$ (83); 67,6\% (69) com idade entre 17 e 20 anos, $89,3 \%$ (91) se declararam solteiros; 80,4\% (82) referem ter concluído o ensino médio em escola privada. O nível econômico dos acadêmicos é privilegiado quando considerada a renda familiar mensal brasileira, em que 54\% (55) da amostra referiu ganho acima de $\mathrm{R} \$ 8.800,00$ (tabela 1 ).

A segunda parte dos resultados envolveu as questões do conhecimento dos motivos que levaram os graduandos a escolher o curso de Odontologia, sendo aceitas no questionário mais de duas opções. A opção mais marcada $(61,7 \%)$ (63) referiu-se à resposta "por ser um curso da área de saúde". A perspectiva após formação acadêmica quanto ao início das atividades profissionais mostrou que $47 \%$ (48) optaram pelo setor público e por montar consultório próprio. Após a conclusão da graduação, $70,5 \%$ (72) dos alunos gostariam de fazer uma especialização para melhor qualificação. Quando abordados a respeito do salário pretendido após 5 anos de formados, 52,9\% (54) pretendiam ganhar entre $\mathrm{R} \$ 4.401,00$ e R \$ 7.040,00 (tabela 2).

Quando questionados com relação à expectativa do curso de Odontologia, 34,3\% (35) responderam para "melhorar a prática atual". Dos entrevistados, 96,0\% (98) marcaram a opção "acham que estão sendo preparados para mercado de trabalho"; e 97,0\% (99) se declararam "satisfeitos com a escolha pela Odontologia" (tabela 3).

Mais da metade dos entrevistados $(62,8 \%)$ (64) mostrou-se satisfeita com o corpo docente da instituição. Referente à visão dos graduandos sobre iniciação científica e/ou monitoria, 44,1\% (45) relataram que pretendem realizar. Com relação à percepção sobre a atuação na Odontologia, foi possível responder a mais de uma alternativa, e 67,6\% (69) escolheram "prevenção e manutenção da saúde". Quando questionados sobre quais disciplinas deveriam ser excluídas ou incluídas no Curso, $72,5 \%$ (74) mostraram-se satisfeitos com a matriz curricular oferecida (tabela 4). 
Tabela 1. Análise descritiva das variáveis Sócio demográficas dos participantes da pesquisa

\begin{tabular}{|c|c|c|c|c|c|c|c|c|c|c|c|}
\hline \multirow{3}{*}{$\begin{array}{c}\text { Variável } \\
\text { Sociodemográfica }\end{array}$} & \multicolumn{10}{|c|}{ Período } & \multirow{3}{*}{ P-Valor } \\
\hline & \multicolumn{2}{|c|}{$2^{\circ}$} & \multicolumn{2}{|r|}{$4^{\circ}$} & \multicolumn{2}{|r|}{$6^{0}$} & \multicolumn{2}{|r|}{$8^{\circ}$} & \multicolumn{2}{|c|}{ Total } & \\
\hline & $\mathbf{n}$ & $\%$ & $\mathbf{n}$ & $\%$ & $\mathbf{n}$ & $\%$ & $\mathbf{n}$ & $\%$ & n & $\%$ & \\
\hline \multicolumn{12}{|l|}{ Gênero } \\
\hline Masculino & 04 & $16 \%$ & 04 & $15,4 \%$ & 06 & $23 \%$ & 05 & $20 \%$ & 19 & $18,6 \%$ & 0,8894 \\
\hline Feminino & 21 & $84 \%$ & 22 & $84,6 \%$ & 20 & $77 \%$ & 20 & $80 \%$ & 83 & $81,4 \%$ & \\
\hline Total & 25 & $100 \%$ & 26 & $100 \%$ & 26 & $100 \%$ & 25 & $100 \%$ & 102 & $100 \%$ & \\
\hline \multicolumn{12}{|l|}{ Idade } \\
\hline Entre 17 a 20 & 20 & $80 \%$ & 22 & $84,6 \%$ & 16 & $61,6 \%$ & 11 & $44 \%$ & 69 & $67,6 \%$ & \\
\hline Entre 21 a 25 & 02 & $08 \%$ & 02 & $7,7 \%$ & 05 & $19,2 \%$ & 13 & $52 \%$ & 22 & $21,6 \%$ & 0,0067 \\
\hline$\geq 26$ & 02 & $08 \%$ & 00 & $00 \%$ & 02 & $7,7 \%$ & 01 & $04 \%$ & 05 & $4,9 \%$ & \\
\hline Não respondeu & 01 & $04 \%$ & 02 & $7,7 \%$ & 03 & $11,5 \%$ & 0 & $00 \%$ & 06 & $5,9 \%$ & \\
\hline Total & 25 & $100 \%$ & 26 & $100 \%$ & 26 & $100 \%$ & 25 & $100 \%$ & 102 & $100 \%$ & \\
\hline \multicolumn{12}{|l|}{ Estado Civil } \\
\hline Solteiro & 23 & $92 \%$ & 20 & $77 \%$ & 25 & $96,2 \%$ & 23 & $92 \%$ & 91 & $89,3 \%$ & 0,8004 \\
\hline Casado & 01 & $04 \%$ & 04 & $15,4 \%$ & 01 & $3,8 \%$ & 01 & $04 \%$ & 07 & $6,9 \%$ & \\
\hline Separado & - & - & 01 & $3,8 \%$ & - & - & - & - & 01 & $01 \%$ & \\
\hline Viúvo & - & - & 01 & $3,8 \%$ & - & - & - & - & 01 & $01 \%$ & \\
\hline Não respondeu & 01 & $04 \%$ & - & - & - & - & 01 & $04 \%$ & 02 & $1,8 \%$ & \\
\hline Total & 25 & $100 \%$ & 26 & $100 \%$ & 26 & $100 \%$ & 25 & $100 \%$ & 102 & $100 \%$ & \\
\hline \multicolumn{12}{|l|}{ Cursou Ensino Médio } \\
\hline Somente em escola pública & 03 & $12 \%$ & 01 & $04 \%$ & 02 & $08 \%$ & 02 & $08 \%$ & 08 & $7,8 \%$ & 0,7026 \\
\hline Somente em escola privada & 19 & $76 \%$ & 21 & $80 \%$ & 20 & $76 \%$ & 22 & $88 \%$ & 82 & $80,4 \%$ & \\
\hline Maior parte em escola pública & - & - & - & - & - & - & 01 & $04 \%$ & 01 & $1,0 \%$ & \\
\hline Maior parte em escola privada & 02 & $08 \%$ & 02 & $08 \%$ & 02 & $08 \%$ & - & - & 06 & $5,9 \%$ & \\
\hline Metade em ambas & 01 & $04 \%$ & 01 & $04 \%$ & 02 & $08 \%$ & - & - & 04 & $3,9 \%$ & \\
\hline Não respondeu & - & - & 01 & $04 \%$ & 00 & $00 \%$ & - & - & 01 & $1,0 \%$ & \\
\hline Total & 25 & $100 \%$ & 26 & $100 \%$ & 26 & $100 \%$ & 25 & $100 \%$ & 102 & $100 \%$ & \\
\hline \multicolumn{12}{|l|}{ Renda Mensal } \\
\hline Até R\$ 880,00 & - & - & 04 & $15,4 \%$ & - & - & - & - & 04 & $3,9 \%$ & \\
\hline De $R \$ 881,00$ a $2.640,00$ & 02 & $08 \%$ & 01 & $3,8 \%$ & 01 & $3,8 \%$ & 02 & $08 \%$ & 06 & $5,9 \%$ & 0,2631 \\
\hline De $R \$ 2.641,00$ a $6.160,00$ & 02 & $08 \%$ & 02 & $7,7 \%$ & 03 & $11,5 \%$ & 04 & $16 \%$ & 11 & $10,8 \%$ & \\
\hline De $R \$ 6.161,00$ a $8.800,00$ & 08 & $32 \%$ & 04 & $15,4 \%$ & 08 & $30,8 \%$ & 04 & $16 \%$ & 24 & $23,6 \%$ & \\
\hline Acima de $\mathrm{R} \$ 8.800,00$ & 13 & $52 \%$ & 13 & $50 \%$ & 14 & $53,9 \%$ & 15 & $60 \%$ & 55 & $54 \%$ & \\
\hline Não respondeu & - & - & 02 & $7,7 \%$ & - & - & - & - & 02 & $1,8 \%$ & \\
\hline Total & 25 & $100 \%$ & 26 & $100 \%$ & 26 & $100 \%$ & 25 & $100 \%$ & 102 & $100 \%$ & \\
\hline
\end{tabular}


Tabela 2. Análise das variáveis referentes à escolha do curso, previsão de início das atividades como profissional, formação continuada e pretensão de salário depois de formado

\begin{tabular}{|c|c|c|c|c|c|c|c|c|c|c|c|}
\hline \multirow{3}{*}{ Variáveis } & \multicolumn{10}{|c|}{ Período } & \multirow{3}{*}{ P-valor } \\
\hline & \multicolumn{2}{|r|}{$2^{\mathbf{0}}$} & \multicolumn{2}{|r|}{$4^{\circ}$} & \multicolumn{2}{|r|}{$\mathbf{6}^{\mathbf{0}}$} & \multicolumn{2}{|r|}{$\mathbf{8}^{\circ}$} & \multicolumn{2}{|c|}{ Total } & \\
\hline & $\mathbf{n}$ & $\%$ & $\mathbf{n}$ & $\%$ & $\mathbf{n}$ & $\%$ & $\mathbf{n}$ & $\%$ & $\mathbf{n}$ & $\%$ & \\
\hline \multicolumn{12}{|l|}{ Motivo da escolha do curso } \\
\hline Vocação & 05 & $4,9 \%$ & 04 & $3,9 \%$ & 04 & $3,9 \%$ & 15 & $14,7 \%$ & 28 & $27,4 \%$ & 0,2856 \\
\hline Compensação financeira & 01 & $0,9 \%$ & 05 & $4,9 \%$ & 05 & $4,9 \%$ & 05 & $4,9 \%$ & 16 & $15,6 \%$ & \\
\hline Profissão liberal & 03 & $2,9 \%$ & 04 & $3,9 \%$ & 13 & $12,7 \%$ & 05 & $4,9 \%$ & 25 & $24,5 \%$ & \\
\hline Existência de faculdade local & - & - & - & - & 01 & $0,9 \%$ & 01 & $0,9 \%$ & 02 & $1,9 \%$ & \\
\hline Influência familiar & 02 & $1,9 \%$ & 05 & $4,9 \%$ & 06 & $5,9 \%$ & 02 & $1,9 \%$ & 15 & $14,7 \%$ & \\
\hline Ser da área de saúde & 15 & $14,7 \%$ & 18 & $17,6 \%$ & 13 & $12,7 \%$ & 17 & $16,7 \%$ & 63 & $61,7 \%$ & \\
\hline $\begin{array}{l}\text { Influência de dentistas parentes/ } \\
\text { amigos }\end{array}$ & 06 & $5,9 \%$ & 08 & $7,8 \%$ & 10 & $9,8 \%$ & 06 & $5,9 \%$ & 30 & $29,4 \%$ & \\
\hline Outros & 01 & $0,9 \%$ & - & - & - & - & - & - & 01 & $0,9 \%$ & \\
\hline \multicolumn{12}{|c|}{ Previsão de Início das atividades Profissionais } \\
\hline $\begin{array}{l}\text { Assalariado somente do setor } \\
\text { público }\end{array}$ & 02 & $1,9 \%$ & 00 & $00 \%$ & 02 & $1,9 \%$ & 03 & $2,9 \%$ & 07 & $6,8 \%$ & \\
\hline $\begin{array}{l}\text { Assalariado somente do setor } \\
\text { particular }\end{array}$ & 07 & $6,8 \%$ & 09 & $8,8 \%$ & 08 & $7,8 \%$ & 15 & $14,7 \%$ & 39 & $38.2 \%$ & 1,0000 \\
\hline $\begin{array}{l}\text { Assalariado do Setor Público e } \\
\text { montar consultório próprio }\end{array}$ & 14 & $13,7 \%$ & 14 & $13,7 \%$ & 15 & $14,7 \%$ & 05 & $4,9 \%$ & 48 & $47 \%$ & \\
\hline Trabalhar em consultório alugado & 02 & $1,9 \%$ & 02 & $1,9 \%$ & 01 & $0,9 \%$ & 02 & $1,9 \%$ & 07 & $6,8 \%$ & \\
\hline $\begin{array}{l}\text { Não pretendo trabalhar em } \\
\text { Odontologia }\end{array}$ & - & - & - & - & - & - & - & - & - & - & \\
\hline Não respondeu & - & - & 01 & $0,9 \%$ & - & - & - & - & 01 & $0,9 \%$ & \\
\hline \multicolumn{12}{|c|}{ O que pretende fazer após a conclusão da graduação } \\
\hline Aperfeiçoamento & - & - & - & - & - & - & 04 & $3,9 \%$ & 04 & $3,9 \%$ & 0,1385 \\
\hline Especialização & 16 & $15,6 \%$ & 20 & $19,6 \%$ & 21 & $20,5 \%$ & 15 & $14,7 \%$ & 72 & $70,5 \%$ & \\
\hline Mestrado e/ou Doutorado & 08 & $7,8 \%$ & 04 & $3,9 \%$ & 04 & $3,9 \%$ & 04 & $3,9 \%$ & 20 & $19,6 \%$ & \\
\hline Estágio & 01 & $0,9 \%$ & 02 & $1,9 \%$ & 01 & $0,9 \%$ & 02 & $1,9 \%$ & 06 & $5,8 \%$ & \\
\hline Não pretende fazer nada & - & - & - & - & - & - & - & - & - & - & \\
\hline \multicolumn{12}{|c|}{ Após 5 anos de formado, qual o salário você imagina estar recebendo } \\
\hline Até $\mathrm{R} \$ 2.640,00$ & 01 & $0,9 \%$ & 01 & $0,9 \%$ & - & - & - & - & 02 & $1,9 \%$ & 0,1562 \\
\hline Entre $\mathrm{R} \$ 2.641,00$ e $\mathrm{R} \$ 4.400,00$ & 01 & $0,9 \%$ & 02 & $1,9 \%$ & 03 & $2,9 \%$ & 01 & $0,9 \%$ & 07 & $6,8 \%$ & \\
\hline Entre $\mathrm{R} \$ 4.401,00$ e $\mathrm{R} \$ 7.040,00$ & 12 & $11,7 \%$ & 20 & $19,6 \%$ & 06 & $5,9 \%$ & 16 & $5,6 \%$ & 54 & $52,9 \%$ & \\
\hline Entre $\mathrm{R} \$ 7.041,00$ e $\mathrm{R} \$ 8.800,00$ & 08 & $7,8 \%$ & 02 & $1,9 \%$ & 09 & $8,8 \%$ & 05 & $4,9 \%$ & 24 & $23,5 \%$ & \\
\hline Mais de $\mathrm{R} \$ 8.801,00$ & 03 & $2,9 \%$ & 01 & $0,9 \%$ & 08 & $7,8 \%$ & 03 & $2,9 \%$ & 15 & $14,7 \%$ & \\
\hline Total & 25 & $100 \%$ & 26 & $100 \%$ & 26 & $100 \%$ & 25 & $00 \%$ & 102 & $100 \%$ & \\
\hline
\end{tabular}


Tabela 3 - Análise referente à percepção dos alunos quanto à expectativa do Curso

\begin{tabular}{|c|c|c|c|c|c|c|c|c|c|c|c|}
\hline \multirow{3}{*}{ Variáveis } & \multicolumn{10}{|c|}{ Período } & \multirow{3}{*}{ P-valor } \\
\hline & \multicolumn{2}{|c|}{$\mathbf{2}^{\mathbf{0}}$} & \multicolumn{2}{|r|}{$\mathbf{4}^{\mathbf{0}}$} & \multicolumn{2}{|r|}{$\mathbf{6}^{\mathbf{0}}$} & \multicolumn{2}{|r|}{$8^{\mathbf{0}}$} & \multicolumn{2}{|c|}{ Total } & \\
\hline & $\mathbf{n}$ & $\%$ & $\mathbf{n}$ & $\%$ & $\mathbf{n}$ & $\%$ & $\mathbf{n}$ & $\%$ & n & $\%$ & \\
\hline \multicolumn{12}{|c|}{ O que você espera da formação no Curso de Odontologia } \\
\hline Preparo para o trabalho & 09 & $8,8 \%$ & 08 & $7,8 \%$ & 09 & $8,8 \%$ & 07 & $6,8 \%$ & 33 & $32.3 \%$ & 0,2708 \\
\hline Cultura geral ampla & 08 & $7,8 \%$ & 07 & $6,8 \%$ & 05 & $4,9 \%$ & 13 & $12,7 \%$ & 33 & $32,3 \%$ & \\
\hline Melhor prática atual & 11 & $10,7 \%$ & 10 & $9,8 \%$ & 04 & $3,9 \%$ & 10 & $9,8 \%$ & 35 & $34,3 \%$ & \\
\hline > Grau de instrução & 08 & $7,8 \%$ & 07 & $6,8 \%$ & 03 & $2,9 \%$ & 10 & $9,8 \%$ & 28 & $27,5 \%$ & \\
\hline Preparo para pesquisa & 03 & $2,9 \%$ & 04 & $3,9 \%$ & 03 & $2,9 \%$ & 07 & $6,8 \%$ & 17 & $16,7 \%$ & \\
\hline $\begin{array}{l}\text { Melhoria da situação } \\
\text { profissional atual }\end{array}$ & 02 & $1,9 \%$ & 04 & $3,9 \%$ & 02 & $1,9 \%$ & 07 & $6,8 \%$ & 15 & $14,7 \%$ & \\
\hline $\begin{array}{l}\text { Conhecimento para melhor } \\
\text { compreender o mundo }\end{array}$ & 02 & $1,9 \%$ & 04 & $3,9 \%$ & 01 & $0,9 \%$ & 09 & $8,8 \%$ & 16 & $15,6 \%$ & \\
\hline $\begin{array}{l}\text { Possibilidade de melhores } \\
\text { salários }\end{array}$ & 03 & $2,9 \%$ & 03 & $2,9 \%$ & 07 & $6,8 \%$ & 06 & $5,9 \%$ & 19 & $18,6 \%$ & \\
\hline Não sabe informar & 02 & $1,9 \%$ & 03 & $2,9 \%$ & 05 & $4,9 \%$ & 00 & $00 \%$ & 10 & $9,8 \%$ & \\
\hline Preparo para o trabalho & 09 & $8,8 \%$ & 08 & $7,8 \%$ & 09 & $8,8 \%$ & 07 & $6,8 \%$ & 33 & $32.3 \%$ & \\
\hline \multicolumn{12}{|c|}{ Você acha que está sendo bem preparado para o mercado de trabalho } \\
\hline Sim & 25 & $24,5 \%$ & 23 & $22,5 \%$ & 26 & $25,4 \%$ & 24 & $23,5 \%$ & 98 & $96 \%$ & 0,1334 \\
\hline Não & - & - & 03 & $2,9 \%$ & - & - & 01 & $0,9 \%$ & 04 & $3,9 \%$ & \\
\hline \multicolumn{12}{|c|}{ Você está satisfeito com sua escolha? } \\
\hline $\operatorname{Sim}$ & 25 & $24,5 \%$ & 24 & $23,5 \%$ & 26 & $25,4 \%$ & 24 & $23,5 \%$ & 99 & $97 \%$ & 0,311 \\
\hline Não & - & - & 02 & $1,9 \%$ & - & - & 01 & $0,9 \%$ & 03 & $2,9 \%$ & \\
\hline Total & 25 & $100 \%$ & 26 & $100 \%$ & 26 & $100 \%$ & 25 & $100 \%$ & 102 & $100 \%$ & \\
\hline
\end{tabular}

\section{DISCUSSÃO}

Atualmente, a questão da feminização na Odontologia está sendo bem discutida. Neste estudo, os resultados apontam que $81,4 \%$ (83) da amostra era do sexo feminino, o que está de acordo com dados do Conselho Regional de Odontologia de São Paulo, que informa que $51,0 \%$ dos dentistas inscritos são mulheres; e entre os que estão com registro provisório, a diferença é ainda maior, $67,0 \%$ são do sexo feminino ${ }^{13}$. No estudo de Oliveira et al. ${ }^{13}$, apenas $7,5 \%$ da amostra possuía renda familiar acima de $\mathrm{R} \$ 6.000$, diferentemente deste estudo, no qual a maioria tinha renda considerada alta, acima desse padrão. Esses resultados ficaram bem próximos, corroborando os estudos realizados pela Universidade Federal do Rio Grande do Sul, no qual, entre os 88 acadêmicos entrevistados, também prevaleceu o sexo feminino $-75,0 \%$ da amostra -, idade entre 17 e 22 anos (89,2\%), solteiros $(91,7 \%)$, cursaram o ensino médio em sua maioria em escola privada e renda familiar de 10 salários mínimos ${ }^{14}$, resultados esses ratificados pelos dados desta pesquisa.

De acordo com este estudo, a maioria dos participantes estudou em escolas privadas, o que está em consonância com os estudos de Santos et al. ${ }^{15}$, realizado com 94 alunos do Curso de Odontologia da Universidade de São Paulo (FOUSP), relatando que a maioria era do sexo feminino, com idade entre 17 e 27 anos, mesma característica encontrada nesta pesquisa. Em todos os estudos que embasaram esses resultados, observou-se a prevalência do sexo feminino. Segundo dados do Plano Nacional de Qualificação, do Ministério do Trabalho e Previdência Social, as mulheres são maioria nas escolas, universidades e cursos de qualificação ${ }^{16}$. A partir dos anos 1970, transformações expressivas aconteceram no perfil 
Tabela 4. Demonstrativo referente à percepção do acadêmico de Odontologia sobre: corpo docente, iniciativa cientifica e ou Monitoria, principal finalidade na Odontologia

\begin{tabular}{|c|c|c|c|c|c|c|c|c|c|c|c|}
\hline \multirow{3}{*}{ Variáveis } & \multicolumn{10}{|c|}{ Período } & \multirow{3}{*}{ P-valor } \\
\hline & \multicolumn{2}{|r|}{$\mathbf{2}^{\mathbf{o}}$} & \multicolumn{2}{|r|}{$4^{\circ}$} & \multicolumn{2}{|r|}{$6^{0}$} & \multicolumn{2}{|r|}{$\mathbf{8}^{\circ}$} & \multicolumn{2}{|c|}{ Total } & \\
\hline & $\mathbf{n}$ & $\%$ & $\mathbf{n}$ & $\%$ & $\mathbf{n}$ & $\%$ & $\mathbf{n}$ & $\%$ & $\mathbf{n}$ & $\%$ & \\
\hline \multicolumn{12}{|c|}{ Avaliação do Corpo Docente } \\
\hline Ótimo & 19 & $76 \%$ & 14 & 53,8 & 15 & 57,7 & 16 & $64,0 \%$ & 64 & $62,8 \%$ & 1,0000 \\
\hline Muito Bom & 06 & $24 \%$ & 10 & 38,5 & 10 & 38,5 & 09 & $36,0 \%$ & 35 & $34,4 \%$ & \\
\hline Bom & - & - & 02 & 7,7 & - & - & - & - & 02 & $1,9 \%$ & \\
\hline Regular & - & - & - & - & 01 & 3,8 & - & - & 01 & $0,9 \%$ & \\
\hline Ruim & - & - & - & - & - & - & - & - & - & - & \\
\hline Total & 25 & $100 \%$ & 26 & $100 \%$ & 26 & $100 \%$ & 25 & $100 \%$ & 102 & $100 \%$ & \\
\hline \multicolumn{12}{|c|}{ Iniciação Cientifica e/ou Monitoria } \\
\hline Pretende realizar & 23 & $92 \%$ & 12 & $46,1 \%$ & 09 & $34,7 \%$ & 01 & $04 \%$ & 45 & $44,1 \%$ & 0,0001 \\
\hline Já realizo com bolsa & - & - & 04 & $15,4 \%$ & 05 & $19,2 \%$ & 09 & $36 \%$ & 18 & $17,6 \%$ & \\
\hline Já realizei & - & - & - & - & - & - & 06 & $24 \%$ & 06 & $5,9 \%$ & \\
\hline Não pretendo realizar & - & - & - & - & 02 & $7,7 \%$ & - & - & 02 & $1,9 \%$ & \\
\hline Gostaria de realizar & 01 & $04 \%$ & 04 & $15,4 \%$ & 05 & $19,2 \%$ & 04 & $16 \%$ & 14 & $13,8 \%$ & \\
\hline Já realizo sem bolsa & 01 & $04 \%$ & 06 & $23,4 \%$ & 05 & $19,2 \%$ & 05 & $20 \%$ & 17 & $16,7 \%$ & \\
\hline Total & 25 & $100 \%$ & 26 & $100 \%$ & 26 & $100 \%$ & 25 & $100 \%$ & 102 & $100 \%$ & \\
\hline \multicolumn{12}{|c|}{ Principal finalidade de cursar Odontologia* } \\
\hline $\begin{array}{l}\text { Prevenção e manutenção } \\
\text { da Saúde Bucal }\end{array}$ & 16 & $15,4 \%$ & 14 & $13,7 \%$ & 17 & $16,7 \%$ & 22 & $21,6 \%$ & 69 & $67,6 \%$ & 0,2835 \\
\hline Prevenção e Tratamento & 17 & $16,7 \%$ & 14 & $13,7 \%$ & 14 & $13,7 \%$ & 14 & $13,7 \%$ & 59 & $57,8 \%$ & \\
\hline Servir à Comunidade & 09 & $8,8 \%$ & 01 & $01 \%$ & 09 & $08,8 \%$ & 08 & $07,8 \%$ & 27 & $26,5 \%$ & \\
\hline Tratar as Doenças & 06 & $06 \%$ & 11 & $10,8 \%$ & 12 & 11,8 & 14 & $13,7 \%$ & 43 & $42,1 \%$ & \\
\hline $\begin{array}{l}\text { Promover Higiene e } \\
\text { Estética }\end{array}$ & 13 & $12,7 \%$ & 14 & $13,7 \%$ & 13 & $12,7 \%$ & 18 & $17,6 \%$ & 58 & $56,9 \%$ & \\
\hline Ser Bem Remunerado & 01 & $01 \%$ & 03 & $2,9 \%$ & 07 & $06,8 \%$ & 08 & $07,8 \%$ & 19 & $18,6 \%$ & \\
\hline Total $*$ & 62 & $60,8 \%$ & 57 & $55,9 \%$ & 72 & $70,6 \%$ & 84 & $\mathbf{8 2 , 3 \%}$ & & & \\
\hline \multicolumn{12}{|c|}{ Das matérias cursadas, alguma deve ser excluída do currículo? } \\
\hline Sim & 01 & $04 \%$ & 13 & $50 \%$ & 19 & $73,1 \%$ & 14 & $56 \%$ & 47 & $46 \%$ & 0,0007 \\
\hline Não & 23 & $92 \%$ & 13 & $50 \%$ & 07 & $26,9 \%$ & 11 & $44 \%$ & 54 & $53 \%$ & \\
\hline Não respondeu & 01 & $04 \%$ & - & - & - & - & - & - & 01 & $01 \%$ & \\
\hline Total & 25 & $100 \%$ & 26 & $100 \%$ & 26 & $100 \%$ & 25 & $100 \%$ & 102 & $100 \%$ & \\
\hline \multicolumn{12}{|c|}{ Alguma matéria deve ser incluída do currículo? } \\
\hline $\operatorname{Sim}$ & 02 & $08 \%$ & 01 & $3,8 \%$ & 04 & $15,4 \%$ & 08 & $32 \%$ & 15 & $14,8 \%$ & 0,0176 \\
\hline Não & 21 & $84 \%$ & 24 & $92,4 \%$ & 17 & $65,4 \%$ & 12 & $48 \%$ & 74 & $72,5 \%$ & \\
\hline Não respondeu & 02 & $08 \%$ & 01 & $3,8 \%$ & 05 & $19,2 \%$ & 05 & $20 \%$ & 13 & $12,7 \%$ & \\
\hline Total & 25 & $100 \%$ & 26 & $100 \%$ & 26 & $100 \%$ & 25 & $100 \%$ & 102 & $100 \%$ & \\
\hline
\end{tabular}

*Mais de uma resposta atribuída

das atividades das mulheres, o que resultou na necessidade econômica e nas oportunidades ofertadas pelo mercado de trabalho. As mudanças demográficas sociais e culturais ocorridas no país acabaram afetando as mulheres, dando-lhes o acesso às universidades, o que, consequentemente, abriu espaços a novas perspectivas de trabalho.

Em relação à percepção dos alunos sobre a 
escolha pelo curso e sobre a proximidade com a área de estudo desejada, a forte tendência para a especialização ficou evidente, realidade essa em alta no mundo devido à globalização, em que o mercado de trabalho exige cada dia mais profissionais especializados e a procura por tecnologia avançada leva os recém-formados à busca de capacitações. Estudo de Mattos e Tenório ${ }^{17}$ com acadêmicos de Odontologia constataram que o aperfeiçoamento técnico-científico e o esforço pessoal foram vistos como determinantes do sucesso profissional, sendo a especialização e o trabalho autônomo apontados como uma das expectativas de futuro entre a maioria dos pesquisados.

Devido ao elevado número de profissionais no mercado de trabalho autônomo, principalmente nos grandes centros, há uma busca dos egressos pelo trabalho formal, porém, montar seu próprio negócio ainda faz parte do objetivo do profissional de odontologia, resultado este claramente apresentado nesta pesquisa, em que a maioria relatou desejar trabalhar como autônomo; e também pela possibilidade de ótimo retorno financeiro ${ }^{15}$. Em contrapartida, os estudos de Freire et al. ${ }^{18}$ revelaram que os conceitos pessoais sobre a Odontologia foram responsáveis pela opção de trabalho autônomo.

Nesta pesquisa, os acadêmicos se mostraram satisfeitos com a matriz curricular, resultados estes também apresentados por Medeiros et al. ${ }^{19} \mathrm{em}$ seu estudo, no qual identificaram que a maioria dos estudantes de Odontologia está satisfeita com o seu desempenho até o momento. Segundo Lazzarin, Nakama e Cordomi Jr. ${ }^{20}$, como em qualquer curso superior, a qualidade do ensino de Odontologia está relacionada com um adequado modelo pedagógico da universidade e do curso. Além disso, a qualificação e a atualização permanente (tanto técnica quanto didático-pedagógica) do corpo docente são essenciais para proporcionar uma formação generalista, humanista, crítica e reflexiva. Neste estudo, verificou-se que os estudantes pesquisados estão satisfeitos com o corpo docente.

Em muitas instituições, a monitoria acadêmica tem-se mostrado como uma excelente opção para complementar e melhorar o processo de ensino-aprendizagem nos cursos de nível superior, haja vista que essa atividade traz grande responsabilidade no processo de socialização na docência universitária, assim como contribui para a qualidade da formação profissional oferecida em todas as áreas. A monitoria acadêmica representa um espaço de formação para o monitor, além de ser uma ação que visa contribuir para a melhoria da qualidade do ensino de graduação, sendo, portanto, de grande valia para a formação dos futuros profissionais $^{21}$. Referente a essa questão, os resultados deste estudo demonstraram que a maior parte dos entrevistados almeja fazer especialização e/ou monitoria. Oliveira e Coelho ${ }^{22}$ ressaltaram que as ações voltadas para o tripé ensino-pesquisaextensão devem ser construídas em ação intersetorial, envolvendo profissionais e acadêmicos em propostas que fortaleçam a formação e ações no serviço com vistas à promoção da saúde.

Relacionado à variável opinião do aluno sobre a grade curricular, a maioria dos acadêmicos entrevistados disse estar satisfeita, porém não se encontraram resultados semelhantes ou que se contraponham aos da presente pesquisa nos estudos referenciados. Não obstante, na pesquisa de Saliba et al. ${ }^{23}$ observou-se que a questão de infraestrutura depende de investimentos na adaptação da grade curricular, os quais vêm sendo feitos nas universidades públicas e privadas brasileiras nos últimos anos, tendo em vista o aprimoramento do ensino. Os autores ressaltam que a maioria das universidades pauta seus ensinamentos em evidências científicas, professores qualificados e disciplinas que respaldem o profissional, o que traz reconhecimento à universidade.

Considerou-se um fator limitante desta pesquisa: a falta de validade externa, não se estendendo para a população de estudantes de outras 
instituições.

$\mathrm{O}$ estudo mostrou que a Odontologia enquanto profissão escolhida pelos acadêmicos entrevistados tem intensa relação com os desejos dos alunos para seu futuro. Acredita-se ser importante conhecer o perfil dos estudantes de Odontologia, pois, assim, pode-se investir mais na formação, na boa infraestrutura e na reputação de uma universidade, uma vez que pesquisas como esta contribuirão para incorporar propriedades importantes para o bom desempenho do curso pela comunidade acadêmica.

\section{CONCLUSÃO}

Conclui-se que os participantes foram motivados a cursar Odontologia por coadunar com seus desejos de atuar na área das Ciências da Saúde, bem como pelo fato de a profissão propiciar um trabalho informal, permitindo um complemento salarial, tendo em vista o empreendedorismo como foco principal.

\section{ABSTRACT \\ Profile of students entering Dentistry school: reasons for choosing it}

The aim of the present cross-sectional, quantitative study was to evaluate the profile of students on the Dentistry course of a private college in Campinas (São Paulo) and their perception of and reasons for choosing such a course. Of the 153 students enrolled in 2016, 102 (66.7\%) agreed to participate in the study. The research tool was a structured, self-administered questionnaire featuring 22 questions that dealt with socioeconomic and demographic profile, perception of the course and reasons for choosing it. Of those who responded, $81.4 \%$ were female. The 17 to 20 year-old age group predominated $(67.3 \%)$, while most of the sample were single $(89.3 \%)$, had attended a private high school (80.4\%), and had a family income of over $\mathrm{R} \$ 8,800.00$ (54.0\%). When asked about the reason for choosing the course, $61.7 \%$ said it was because it was part of the area of health, while $29.4 \%$ were influenced by a dentist. Regarding professional intentions, $6.8 \%$ wanted to work in the public sector only, $47.0 \%$ wanted to be salaried employees and set up their own business and 38\% wanted to be self-employed in a dental surgery. A total of $62.8 \%$ declared themselves satisfied with the teaching staff of the course; $72.5 \%$ said they were satisfied with the curriculum, and the majority (70.5\%) planned to do a specialization course after graduation. With regard to their choice, $97.0 \%$ were satisfied; while $96.0 \%$ felt prepared to enter the job market. The perception of the respondents about their training was positive. A trend towards salaried work in the profession was noted, although a desire to work in the private sector was also noted. Descriptors: Dentistry. Choice of Profession. Students.

\section{REFERÊNCIAS}

1. Costa SM, Bonan PRF, Abreu MHNG, Durães SJA. Representação social da Odontologia: uma abordagem qualitativa junto aos graduandos da Unimontes. Rev Odonto Ciência 2008; 23(3):238-42.

2. Silva RHA, Sales-Peres A. Odondologia: um breve histórico. Odontologia Clin-Cientif. 2007; 6(1):7-11.

3. Morita MC, Haddad AE, Araújo ME. Perfil atual e tendências do Cirurgião-dentista brasileiro. Maringá: Dental Press International; 2010.

4. Pereira W. Uma História da odontologia no Brasil. Hist Perspect. 2012;(47):147-73.

5. Souza JES, Maciel LKB, Zocratto KBF. O papel do ensino de graduação em Odontologia e o motivo da escolha da profissão: uma visão dos alunos concluintes. RFO UPF; 18(3):277-83.

6. Ferreira NP, Ferreira AP, Freire MCM. Mercado de trabalho na Odontologia: contextualização e perspectiva. Rev Odontol UNESP. 2013; 42(4):304-09.

7. Bardagi M, Lassance MCP, Paradiso AC, Menezes IA. Escolha Profissional e Inserção no Mercado de Trabalho - Percepções de Estudantes Formandos. Psicol Esc Educ. 
2006;10(1):69-82.

8. Carcereri DL, Amante CJ, Reibnitz MT, Mattevi GS, Silva GG, et al. Formação em Odontologia e interdisciplinaridade: o PróSaúde da UFSC. Rev ABENO. 2011; 11(1):6270.

9. Morita MC, Haddad AE, Araújo ME. Perfil atual e tendências do cirurgião-dentista brasileiro. Maringá: Dental Press, 2010.

10. Brasil. Ministério da Educação e Cultura. Instituições de Educação Superior e Cursos Cadastrados. Curso de Graduação em Odontologia. MEC [Acesso em 14 dez. 2018]. Disponível em: http://emec.mec. gov.br/emec/ nova\#avancada.

11. Colegiado do curso de odontologia da UFBA. Projeto Pedagógico da Faculdade de Odontologia da UFBA. Salvador, BA, 2012. [Acesso em 15 maio. 2018]. Disponível em: https://odo.ufba.br/grad/projeto-pedagógico.

12. Junqueira JC, Colombo CED, Tavares PG, Rocha RF, Carvalho YR, Rodrigues JR. Quem é e o que pensa o graduando de odontologia. Rev Odontol UNESP. 2002; 31(2): 269-84.

13. Oliveira DL, Souza ES, Batista FJN. Perfil do aluno de odontologia da universidade estadual do sudoeste da Bahia - UESB. Rev Saúde Com. 2013; 9(3):169-78.

14. Nauodonto. Núcleo de Avaliação da Unidade. Faculdade de Odontologia. Universidade Federal do Rio Grande do Sul. Projeto perfil dos ingressantes do curso diurno de odontologia da Universidade Federal do Rio Grande do Sul (Resultados parciais - Turma 2014-1 e 2014-2). 2014. [Acesso em 10 set 2016] Disponível em: http://www.ufrgs.br/ odontologia/arquivos/projetos-nau/resumosprojeto-perfil-ingressante-odontologia-diurno.

15. Santos, BRM, Gonzalez PS, Carrer FCA. Perfil e expectativas dos ingressantes da Faculdade de Odontologia da USP: uma visão integrada com as diretrizes curriculares nacionais e o sistema único de saúde. Rev ABENO. 2015; 15(1):28-37.

16. Portal Brasil. Caderno Economia e Emprego. Mulheres são maioria em universidades e cursos de qualificação. 2016. [Acesso em 15 out. 2016]. Disponível em: http://www.brasil. gov.br/economia-e-emprego/2016/03/mulhere s-sao-maioria-em-universidades-e-cursos-dequalificacao.

17. Matos MS, Tenório RM. Expectativas de estudantes de Odontologia sobre o campo de trabalho odontológico e o exercício profissional. Rev Bras Pesq Saúde. 2011; 13(4):10-21.

18. Freire MC, Jordão LM, Ferreira NP, Nunes MF, Queiroz MG et al. Motivation towards career choice of Brazilian freshman students in a fifteen year period. J Dent Educ. 2011; 75(1):115-21.

19. Medeiros CLA, Queiroz MDD, Souza GCA et al. Expectativas de cirurgiões dentistas sobre a inserção da saúde bucal no programa saúde da família. Rev Eletr Enferm. 2007; 9(2):379-88.

20. Lazzarin HC, Nakama L, Cordoni Júnior L. O papel do professor na percepção dos alunos de odontologia. Saúde Soc. 2007; 16(1):90-101.

21. Nunes JBC. Monitoria acadêmica: espaço de formação. Coleção Pedagógica n. 9, 2006.

22. Oliveira ML, Coelho TC. A percepção de acadêmicos de odontologia sobre o PET-Saúde UFMS/ SESAU, Campo Grande/MS, 2009. Rev ABENO. 2011; 11(1):76-80.

23. Saliba NA, Moimaz SAS, Prado RL et al. Percepção do cirurgião-dentista sobre formação profissional e dificuldades de inserção no mercado de trabalho. Rev Odontol UNESP. 2012; 41(5):297-304.

\section{Correspondência para:}

Arlete Maria Gomes Oliveira e-mail: arlete.maria@yahoo.com.br Rua José Rocha Junqueira, 13 - Swift 13045-755 Campinas/SP 